Pteridines

Vol. 13, 2002, pp. $121-125$

\title{
Moderate Hyperhomocysteinemia in Patients with Huntington's Disease
}

\author{
Andreas Laich', Friedrich Leblhuber', Bernhard Widner', Barbara Frick', Kurt Jellinger ${ }^{3}$, Dietmar Fuchs ${ }^{1.4}$
}

'Institute for Medical Chemistry and Biochemistry, Leopold Franzens University, Fritz Preg1 Strasse 3, A-6020 Innsbruck, Austria, 'Department of Gerontology, Neurologic Clinic Wagner-Jauregg, A-4021 Linz, Austria, ${ }^{3}$ Clinic for Neuropathology, Vienna City Hospital, and Ludwig Boltzmann Institute for Neurobiology, Wolkersbergenstrasse 1, A-1130 Vienna, Austria, ${ }^{4}$ Ludwig Boltzmann Institute for AIDS-Research, Fritz Preg1 Strasse 3, A-6020 Innsbruck, Austria

\begin{abstract}
Hyperhomocysteinemia is considered to be an independent risk factor for cardiovascular diseases and atherosclerosis. Also patients with dementia, either of Alzheimer's or of vascular type, may present with elevated homocysteine levels. All these disorders are linked with older age and since hyperhomocysteinemia is also frequent in the healthy elderly, we were interested to determine homocysteine concentrations in a younger population of demented patients suffering from Huntington's disease. In 15 patients with Huntington's disease and in a control group of similar age, serum homocysteine concentrations were measured and changes were compared to concentrations of folate and neopterin, the latter being an indicator of immune activation. A subgroup of patients with Huntington's disease presented with elevated homocysteine concentrations compared to healthy controls of similar age. Moderate hyperhomocysteinemia was related to lower folate concentrations and also to higher neopterin levels which indicate increased immune activation. The correlation of homocysteine with neopterin concentrations points to a possible relationship between the development of hyperhomocysteinemia and immune activation in the patients.
\end{abstract}

Key words: homocysteine, Huntington's disease, neopterin, dementia

\section{Introduction}

The non-protein forming, sulfur-containing amino acid homocysteine has intensively been studied in recent years. Increased homocysteine concentrations can be i) due to genetic defects (i.e. mutations in the genes encoding for the enzymes methioninsyntase or $\mathrm{N}$-5-methylenetetrahydrofolate reductase), ii) due to metabolic dysfunction or iii) due to insufficient availability of vitamins involved in the metabolism, namely folate, $B_{12}$ and $B_{6}$, required for the conversion of homocysteine (6). Elevated homocysteine levels are known as independent risk factor for cardiovascular disease $(1,4)$, and also peripheral arterial disease, venous thrombosis and renal failure are linked to genetic hyperhomocysteinemia. More recently, elevated plasma homocysteine levels were reported in patients with neurodegenerative disorders like Alzheimer's disease $(3,16)$ or vascular dementia (16) and also in Parkinson's disease (17). All these disorders are merely diagnosed in older-aged patients and since hyperhomocysteinemia is also frequent in the healthy elderly, we were interested to determine homocysteine concentrations in a younger population of demented patients with suffering from Iuntington's disease.

In neurodegenerative diseases, markers of immune activation such as neopterin and soluble cytokine receptors were found increased in the peripheral blood and to correlate to disease progression $(14,15,28)$. Besides, a relationship between moderate hyperhomo-

Correspondence to: Dr. Dietmar Fuchs, Institute for Medical Chemistry and Biochemistry, Leopold Franzens University, Fritz Pregl Strasse 3, A-6020 Innsbruck, Austria, Phone +43512 507 3519, Telefax +43 5125072865 E-mail dietmar.fuchs@uibk.ac.at 
cysteinemia and increased neopterin concentrations appears to exist (27) pointing to a role of immune activation and oxidative stress to underlie folate depletion and hyperhomocysteinemia.

In this study, we examined homocysteine concentrations in patients suffering from Huntington's disease, and the concentrations were compared to concomitantly measured concentrations of vitamins folate and $\mathrm{B}_{12}$ and of neopterin.

\section{Materials and Methods}

\section{Subjects}

We investigated a total of 15 patients $(9$ male, 6 female) suffering from Huntington's disease (diagnosis proven by molecular genetics or autopsy, aged $40.9 \pm 12.3$ years (disease duration: 2 - 14 years, mean 7.5 years). There were no clinical signs of or laboratory parameters suggesting infection or nephropathy in the subjects investigated. The study was approved by the local ethic committee, and informed consent was obtained from unaffected relatives of patients with Huntington's disease.

Patients were free of continuous neuroleptic treatment for at least one week, drugs were only given occasionally (10 ggt haloperidol; $12.5 \mathrm{mg}$ clonazepam) for sedation.

\section{Measurements}

Blood specimens from patients were taken in the morning after a nocturnal fasting period, and blood was centrifuged immediately at $3000 \mathrm{rpm}$ for $2 \times 15$ min. Thereafter sera were stored at $-20^{\circ} \mathrm{C}$ until measurement.

Total homocysteine was measured by fluorescence polarization immunoassay (Abbott, Vienna, Austria) according to the manufacturers protocol. Total homocysteine is defined as the sum of all homocysteine species including free and protein-bound mono- and dimeric forms (26). The latter contributing two molecules to the homocysteine level which requires a preceding reduction step in sample preparation.

Folate and vitamin $\mathrm{B}_{12}$ concentrations were determined simultaneously by a double-labelled radioimmunoassay (Magic, No Boil, Chirion Diagnostic corporation, MA, USA). The active isotops were ${ }^{125} \mathrm{I}$ for folate and ${ }^{57} \mathrm{Co}$ for vitamin $\mathrm{B}_{12}$. Neopterin was determined by ELISA (BRAHMS Diagnostica, Berlin, Germany).

Result were compared to a control group with similar sex and age distribution which has been examined earlier (16) with the same pre-analytical treatment of blood specimens.

\section{Statistics}

Because distribution of data was not normal, statistical analyses were performed by non-parametric Spearman rank correlation and by $\mathrm{X}^{2}$-test (statistical software package SPSS for Windows Version 10.0).

\section{Results}

In the patients with Huntington's disease, the median concentrations (interquartile ranges in brackets)

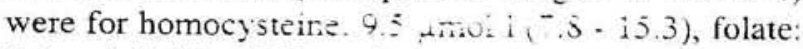
$9.5 \mu \mathrm{g} / 1$ (4.20 - 20.9), and vitamin $B_{12}: 343 \mathrm{ng} / 1$ (277 531). Median neopterin concentration was $6.5 \mu \mathrm{g} / 1$ (5.2 - 11.7). For homocysteine, the normal range is defined as $5-15 \mu \mathrm{mol} / 1$ (13) which agrees well with the concentrations found in our earlier investigation of controls (below $15 \mu \mathrm{mol} / 1$ homocysteine for males and 12 for females (16)) and all controls in this study presented with homocysteine concentrations within these ranges. Compared to this limit, 5 of the 15 patients $\left(33 \% ; X^{2}=4.84, p=0.03, X^{2}\right.$-test $)$ presented with increased homocysteine concentrations. Compared to the lower limit ( $5^{\text {th }}$ percentiles) of serum vitamin concentrations in normal subjects $(11 \mu \mathrm{g} / \mathrm{l}$ for folate and $185 \mathrm{ng} / \mathrm{I}$ for vitamin $\mathrm{B}_{12}$ ) none of the patients had decreased folate concentrations, and 2 patients (13\%) presented with decreased vitamin $\mathrm{B}_{12}$ concentrations. Four patients $(27 \%)$ had increased neopterin concentrations compared to the $95^{\text {th }}$ percentile of healthy controls $=8.7 \mathrm{nmol} / \mathrm{l}(8)$.

An inverse association was found between homocysteine and folate concentrations, and a comparably strong correlation existed between neopterin and homocysteine concentrations (Table 1). There was also a significant correlation between age and neopterin concentrations. No correlations were found between duration of the disease and any of the parameters investigated.

\section{Discussion}

Moderate hyperhomocysteincmia was found in a subgroup of patients suffering from Huntington's disease, and homocysteine concentrations correlated inversely with folate levels. From the data we conclude that also in Huntington's disease, folate deficiency may be responsible for increased serum homocysteine concentrations. No such correlation existed between homocysteine and vitamin $\mathrm{B}_{12}$. However, the number of patients available may be too low to exclude such a relationship. There was no association of homocysteine concentrations with age or duration of disease, but homocysteine correlated strongly with neopterin concentrations, a marker of immune activation. 
Table 1. Spearman rank correlation coefficients (p-values in brackets) between homocysteine, folate, vitamin B12, neopterin and age in 15 patients with Huntington's disease; n.s. = not significant

$\begin{array}{lllll} & \text { Folate } & \text { Vitamin B12 } & \text { Neopterin } & \text { Age } \\ \text { Homocysteine } & -0.735 & -0.239 & 0.645 & 0.320 \\ & (<0.01) & \text { n.s. } & (<0.01) & \text { n.s. } \\ \text { Folate } & & 0.201 & -0.354 & -0.040 \\ & & \text { n.s. } & \text { n.s. } & \text { n.s. } \\ \text { Vitamin B12 } & & 0.190 & 0.311 \\ & & & \text { n.s. } & \text { n.s. } \\ \text { Neopterin } & & & 0.637 \\ & & & & (<0.01)\end{array}$

Since homocysteinuria has been described (9) it became more and more evident that a lack of essential nutritional components like folate or vitamins $B_{6}$ and $B_{12}$ (cobalamin) or the malfunction of enzymes involved in the homocysteine metabolism contributes markedly to the development of hyperhomocysteinemia. When folate and/or vitamins $B_{12}$ availability is reduced, conversion of homocysteine is limited and hyperhomocysteinemia is likely to develop. It is also assumed that inadequate intake of essential cofactors like folate or vitamins $B 6$ and $B_{12}$ may cause accumulation of homocysteine and hyperhomocysteinemia can be at least partly corrected with dietary supplementation of folate or vitamin $B_{12}(11,23)$. Nutritional status of severely demented patients very often is subnormal. But independent from the background of hyperhomocysteinemia, supplementation with folate may also be able to correct it also in patients with Huntington's disease.

Huntingtin was found to interfere with cystathionine- $\beta$-synthase (2), an L-serine hydrolase adding homocysteine, and is thus probably able to interact with homocysteine metabolism. On the other hand in the present study, homocysteine concentrations correlated with neopterin concentrations. Neopterin is a product of monocyte-derived macrophages activated by interferon-g, and neopterin concentrations allow to monitor cell-mediated $=\mathrm{Th}-1$ type immune response in vitro and in vivo (7). Elevated neopterin concentrations in cerebrospinal fluid and blood of patients with neurodegenerative disorders like Alzheimer's disease and Huntington's disease, were found earlier to correlate with other markers of immune activation such as soluble TNF receptors and with cognitive abilities of patients $(14,15)$. Since neopterin production coincides with the formation of toxic products like reactive oxygen species during cellular immune response, increased neopterin concentrations also serve as an estimate of oxidative stress elicited during episodes of immune activation (16). An association exists between higher neopterin concentrations and decreased vitamin $\mathrm{E}$ ( $\alpha$-tocopherol) concentrations in nonagenerians and in patients with dementia $(22,24)$.

Oxidative stress appears to play an important role in the loss of neurons characteristic for neurodegenerative diseases (12), and it has even been claimed that homocysteine production is capable of contributing to oxidative processes (19). The correlation found between neopterin and homocysteine concentrations points to an association between immune system activation, oxidative stress and the development of hyperhomocysteinemia. Only enzymatically reduced 5,6,7,8-tetrahydrofolate, the precursor of methyltetrahydrofolate, is the active folate derivative required for the conversion of homocysteine. Tetrahydrofolates are very susceptible to irreversible oxidation because of degradation and is therefore rapidly destroyed in an oxidative milieu (27). Therefore, folate deficiency may develop in patients under oxidative stress conditions even when dietary supply is within the recommended range. Similar relationships were suggested recently to exist for vitamin $B_{12}$ deficiency in patients with Alzheimer's disease (28).

Our study provides only correlational evidence which cannot confirm a cause-effect relationship. However, similar association between neopterin and homocysteine concentrations has been identified earlier also in other disease states, e.g. in pre-eclampsia and in patients with disturbed glucose metabolism $(10,20)$ and also in Parkinson's disease (29) and in healthy controls (25). Finally a correlation was found between cytokine TNF- $\alpha$ and homocysteine concentrations in patients with atherosclerosis, seropositive for Chlamydia pneumoniae, which also supports the 
notion of an association between immune activation and the accumulation of homocysteine (30).

There were no signs of infection or inflammation in our patients. Thus, the underlying stimulus of immune activation is unclear, but cellular immune activation appears to coincide with hyperhomocysteinemia. Probably the same stimuli which are responsible for oxidative stress and neurodegeneration are involved to trigger the immune system.

In our patients, age correlated with neopterin concentrations. Higher homocysteine and also neopterin concentrations were shown earlier in older aged people as compared to the young $(5,21)$. Correlation found between neopterin levels and age may be related to a longer disease duration in older patients. Statistics did not reveal such a relationship, but again the number of cases may be to low to exclude such an association. Further studies will be necessary to clarify this point.

We conclude that a subgroup of patients with Huntington's disease presents with moderate hyperhomocysteinemia which coincides with cellular immune activation. Oxidative stress could be involved to destroy cofactors necessary for the conversion of homocysteine. Dietary vitamin supplementation may help to overcome possible consequences of enhanced requirement of antioxidant vitamins like folate.

\section{Acknowledgement}

Financial support by the Austrian Funds "Zur Förderung der wissenschaftlichen Forschung", project 14942 is gratefully acknowledged.

\section{References}

1 Boushey CJ, Beresford SA, Omenn GS, Motulsky AG: A quantitative assessment of plasma homocysteine as a risk factor for vascular disease. Probable benefits of increasing folic acid intakes. J Am Med Assoc 1995, 274:1049-1057

2 Boutell JM, Wood JD, Harper PS, Jones AL: Huntingtin interacts with cystathionine beta-synthase. Hum Mol Genet 1998, 7:371-378

3 Clarke R, Smith AD, Jobst KA, Refsum H, Surtton L, Ueland PM: Folate, vitamin B12 and serum total homocysteine levels in confirmed Alzheimer disease. Arch Neurol 1998, 55:1449-1455

4 Eikelboom JW, Lonn E, Genest J, Hankey G, Yusuf $\mathrm{S}$ : Homocyst(e)ine and cardiovascular disease: a critical review of the epidemiologic evidence. Ann Int Med 1999, 131:363-375

5 Fahcy JL, Schnelle JF, Boscardin J, Thomas JK, Gorre ME, Aziz N, Sadeghi H, Nishanian P. Distinct categories of immunologic changes in frail elderly. Mech Ageing Dev 2000, 15:1-20

6 Finkelstein JD: The metabolism of homocysteine: pathways and regulation. Eur J Pediat 1998, $157 ;$ S40-S44

7 Fuchs D, Hausen A, Reibnegger G, Werner ER, Dierich MP, Wachter H: Neopterin as a marker for activated cell-mediated immunity: Application in HIV infection. Immunol Today 1988, 9:150-215

8 Fuchs D, Weiss G, Reibnegger G, Wachter H: The role of neopterin as a monitor of cellular immune activation in transplantation, inflammatory, infectious and malignant diseases. Crit Rev Clin Lab Sci 1992, 29:307-341

9 Gerritson T, Vaughn JG, Waisman HA: Identification of homocystine in urine. Biochim Biophys Res Commun 1962, 9:493-496

10 Gottsater A, Anwaar I, Eriksson KF, Mattiasson I, Lindgarde F: Homocysteine is related to neopterin and endothelin-1 in plasma of subjects with disturbed glucose metabolism and reference subjects. Angiology 2000, 51:489-497

11 Graham IM, O'Callaghan P: The role of folic acid in the prevention of cardiovascular disease. Curr Opin Lipidol 2000, 11:577-587

12 Halliwell B: Oxidants and the central nervous system: some fundamental questions. Is oxidant damage relevant to Parkinson's disease, Alzheimer's disease, traumatic injury or stroke? Acta Neurol Scand 1989; 126:S23-S33

13 Joosten E, van den Berg A, Riezler R, Naurath HJ, Lindenbaum J, Stabler SP, Allen RH: Metabolic evidence that deficiencies of vitamin B12 (cobalamin), folate, and vitamin B6 occur commonly in elderly people. Am J Clin Nutr 1993, 58:468-476

14 Leblhuber F, Walli J, Jellinger K, Tilz GP, Widner B, Laccone F, Fuchs D: Activated Immune system in patients with Huntington's disease. Clin Chem Lab Med 1998, 36:747-750

15 Leblhuber F, Walli J, Demel U, Tilz GP, Widner B, Fuchs D: Increased serum neopterin concentrations in patients with Alzheimer's disease. Clin Chem Lab Med 1999, 37:429-431

16 Leblhuber F, Walli J, Artner-Dworzak E, Vrecko K, Widner B, Reibnegger G, Fuchs D: Hyperhomocysteinemia in dementia. J Neural Transm 2000, 107:1469-1474

17 Müller T, Werne B, Fowler B, Kuhn W: Nigral endothelial dysfunction, homocysteine, and Parkinson's disease. Lancet 1999, 354:126-127

18 Murr C, Fuith LC, Widner B, Wirleitner B, BaierBitterlich G, Fuchs D: Increased neopterin concentrations in patients with cancer: indicator of oxidative stress? Anticancer Res 1999, 19:1721-1728

19 Olszewski AJ, McCully KS: Homocysteine metab- 
olism and the oxidative modification of proteins and lipids. Free Radic Biol Med 1993, 14:683-693

20 Powers RW, Evans RW, Majors AK, Ojimba JI, Ness RB, Crombleholme WR, Roberts JM: Plasma homocysteine concentration is increased in preeclampsia and is associated with evidence of endothelial activation. Am J Obstet Gynecol 1998, 179:1605-1611

21 Reibnegger G, Huber LA, Jürgens G, Schoenitzer D, Werner ER, Wachter $\mathrm{H}$, Wick $\mathrm{G}$, Traill $\mathrm{KN}$ : Approach to define "normal aging" in man. Immune function, serum lipids, lipoproteins and neopterin levels. Mech Ageing Dev 1989; 46:67-82

22 Sattler W, Leblhuber F, Walli J, Widner B, Fuchs $D$ : Cerebrospinal fluid levels of a-tocopherol and neopterin in patients with dementia. Pteridines $1999,10: 220-224$

23 Selhub J, Jacques PF, Rosenberg IH, Rogers G, Bowman BA, Gunter EW, Wright JD, Johnson CL: Serum total homocysteinc concentrations in the third national health and nutrition examination survey (1991-1994): population reference ranges and contribution of vitamin status to high serum concentrations. Ann Int Med 1999, 131:331-339

24 Solichova D, Melichar B, Blaha V, Klejna M, Vavrova J, Palicka V, Zadak Z: Biochemical profile and survival in nonagenarians. Clin Biochem 2001, 34:563-569
25 Turgan N, Habif S, Parildar Z, Ozmen D, Mutaf I, Erdener D, Bayindir O: Association between homocysteine and neopterin in healthy subjects measured by a simple HPLC fluorometric method. Clin Biochem 2001, 34:271-275

26 Ueland PM, Refsum G, Stabler SP, Malinow MR, Andersson A, Allen RH: Total homocysteine in plasma or serum: methods and clinical application. Clin Chem 1993, 39:1964-1979

27 Widner B, Enzinger C, Laich A, Wirleitner B, Fuchs D: Hyperhomocysteinemia pteridines and oxidative stress. Current Drug Metabol 2002, 3:225-232

28 McCaddon A, Regland B, Hudson P, Davies G. Functional vitamin $B(12)$ deficiency and Alzheimer disease. Neurology 2002, 58:1395-1399

29 Widner B, Leblhuber F, Frick B, Laich A, ArtnerDworzak E, Fuchs D. Moderate hyperhomocysteinaemia and immune activation in Parkinson's disease. J Neural Transm 2002;109:1445-1452

30 Wiesli P, Maly FE, Meniconi A, Czerwenka W, Hoffmann U, Vetter W, Schulthess G: Chlamydia pneumoniae seroposivity and hyperhomocysteinemia are linked in patients with atherosclerosis. Clin Chem 2001, 47:1304-1306 